Notfall Rettungsmed 2014 • [jvn]:[afp]-[alp]

DOI 10.1007/s10049-014-1918-8

Online publiziert: 2 . August 2014

(c) Springer-Verlag Berlin Heidelberg 2014
B.W. Böttiger ${ }^{1} \cdot$ C. Waydhas ${ }^{2} \cdot$ C. Wrede ${ }^{3}$

${ }^{1}$ Universitätsklinikum Köln, Köln

2 Universitätsklinikum Essen, Essen

${ }^{3}$ Interdisziplinäres Notfallzentrum, HELIOS Klinikum Berlin-Buch, Berlin

\title{
Notfallmedizin ist interdisziplinär und interprofessionell
}

\author{
Die neue Schriftleitung stellt sich vor
}

Sehr geehrte Leserinnen und Leser, liebe Kolleginnen und Kollegen,

vor wenigen Monaten sind die beiden langjährigen und sehr erfolgreichen Gesamtschriftleiter der Zeitschrift Notfall + Rettungsmedizin, Prof. Dr. Uwe Kreimeier und Prof. Dr. Hans-Richard Arntz aus dieser Funktion ausgeschieden. Beide haben maßgeblich und federführend dazu beigetragen, dass die Zeitschrift Notfall + Rettungsmedizin das geworden ist, was sie heute darstellt: die wichtigste notfallmedizinische Zeitschrift im deutschsprachigen Raum - eine Zeitschrift, die dem wissenschaftlichen Anspruch nach schneller $\mathrm{Pu}$ blikation neuer Erkenntnisse nachkommt, ein sehr breit gefächertes Weiterbildungsangebot anbietet sowie mit Themen zu Organisation, Struktur und Qualität zur positiven Entwicklung der gesamten Notfallmedizin in Deutschland und dem deutschsprachigen Ausland beiträgt. Dabei stehen die Interdisziplinarität, die Interprofessionalität und die Sachbezogenheit immer im Mittelpunkt. Dafür sei Ihnen auch vom neuen Herausgeberteam ganz herzlich gedankt. Das hohe Niveau der Zeitschrift, das erreicht wurde, stellen für uns Verpflichtung, Ansporn und Herausforderung zugleich dar, die Erfolge unserer Vorgänger zu sichern und zu mehren.

Was ist uns jetzt für die nächsten Jahre wichtig? Ganz im Mittelpunkt stehen als erstes alle unsere Leser, und damit natürlich weiterhin Interdisziplinarität und Interprofessionalität, was sich auch im Spektrum unserer beruflichen Herkunft und unserer breiten internen Über- einstimmung über unsere gemeinsamen Ziele für die Zeitschrift widerspiegelt. Die notwendige Vielfältigkeit und Multiprofessionalität der Notfallmedizin und ihrer Protagonisten zeigt sich hier auch bei den Rubrikherausgebern und den Mitgliedern des Editorial Boards. Wichtig ist für uns dabei, dass unsere Zeitschrift mit ihren Themen und Schwerpunkten sowohl die präklinischen Aspekte der Notfallmedizin und des Rettungsdienstes als auch die klinische Notfall- und Akutmedizin in den Notaufnahmen und allen weiteren Bereichen der Krankenhäuser gleichermaßen repräsentiert. Auch wenn die Grundsätze der Notfallversorgung unabhängig davon sind, wo der Notfall auftritt, so ist es doch offensichtlich, dass die unterschiedlichen Strukturen innerhalb und außerhalb des Krankenhauses unterschiedliche Möglichkeiten bieten, unterschiedliche Ressourcen vorhalten und dadurch mitunter auch andere Behandlungspfade und -strategien erfordern.

\section{》) Im Mittelpunkt stehen Interdisziplinarität und Interprofessionalität}

Während der Schwerpunkt der präklinischen Notfallmedizin - und auch auf den Stationen eines Krankenhauses - häufig bei der Erkennung und Stabilisierung kritischer und nicht selten vitaler Patientenzustände liegt, ist der Anteil der kritisch kranken Patienten in den Notaufnahmen unserer Krankenhäuser relativ gering, und Diagnostik und Therapie von Patien- ten mit akuten Erkrankungen nehmen hier einen großen Raum ein. Unser Ziel ist es, dass Notfall + Rettungsmedizin aktuelles medizinisches Wissen und Handlungspfade für alle Bereiche der Notfallmedizin vermittelt und damit weiterhin einen wesentlichen Beitrag zur Verbesserung der notfallmedizinischen Patientenversorgung leistet.

Soll Notfall + Rettungsmedizin auch ein politisches Forum sein? Natürlich ja und nein! "Ja“ in dem Sinne, dass medizinorganisatorische, gesellschafts- und berufspolitische Entwicklungen aufgegriffen und diskutiert werden. Dabei sollen Probleme und ungelöste Fragen angesprochen und insbesondere Informationen und Sachstände vermittelt werden. Wir wollen ausdrücklich, dass die Zeitschrift, wo möglich, zur Problemerkennung und Problemschärfung beiträgt und wichtigen Themen ein Forum gibt. Sie soll zur Verbesserung der notfallmedizinischen Versorgung all unserer Patienten beitragen und eine Diskussion wichtiger Fragen in der Community der professionellen Notfallversorger anregen und fördern. „Nein“ in dem Sinne, dass wir nicht zum politischen Sprachrohr einzelner Fachdisziplinen, Berufsgruppen oder Interessenvertreter werden wollen. Evidenz, Fakten und kollegiale, konstruktive Sachbezogenheit sind und bleiben unsere zentra-

Herr Prof. B.W. Böttiger, Herr Prof. C. Waydhas und Herr PD Dr. C. Wrede sind als Schriftleiter der Notfall + Rettungsmedizin gleichberechtigt an der Gestaltung des Editorials und dessen Inhalt beteiligt gewesen. 
le Maxime. In diesem Sinne sind auch die Seiten unserer Zeitschrift wichtig, die für Verbände und Organisationen reserviert sind, deren Beiträge ebenfalls zur Diskussion und Meinungsbildung beitragen.

Wie wird es weitergehen? Die Bereiche „Originalarbeiten“, „CME“ und „Journal Club“ bleiben neben den anderen $\mathrm{Ru}$ briken die zentralen Pfeiler unserer Zeitschrift und sollen weiter ausgebaut werden. Zusätzlich ist geplant, regelmäßig über technische Innovationen zu berichten, um Ihnen auch Hilfestellung und Orientierung bei der apparativen Ausstattung von Rettungsmitteln, Notaufnahmen, intrahospitalen Notfallteams u. a. Bereichen zu geben. Nur durch das großartige Engagement der Autoren ist eine hohe Qualität der Beiträge und damit der Zeitschrift möglich.

Wo wollen wir hin? Wir werden Ihnen allen weiterhin absolut hochwertige, für Ihren klinischen Alltag und das Wohl Ihrer Patienten hochrelevante und breit gefächerte Beiträge zu den wichtigen Themen der prä- und innerklinischen Notfallmedizin bieten. Wir werden die Attraktivität unserer Zeitschrift für Autoren weiter steigern und die Position der Notfall + Rettungsmedizin als führende notfallmedizinische Zeitschrift für Ärzte, für Rettungsdienstpersonal und für Pflegende im deutschsprachigen Raum sichern und weiter ausbauen.

Wir freuen uns sehr auf unsere gemeinsame Tätigkeit sowie auch auf einen intensiven Kontakt und Austausch mit Ihnen

Ihre

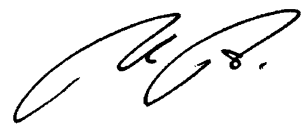

Bernd Böttiger

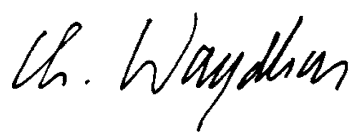

Christian Waydhas

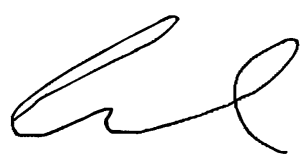

Christian Wrede

\section{Korrespondenzadresse}

\section{Prof. Dr. B.W. Böttiger}

Universitätsklinikum Köln

Köln

bernd.boettiger@uk-koeln.de

Prof. Dr. C. Waydhas

Universitätsklinikum Essen

Essen

Christian.Waydhas@uk-essen.de

\section{PD Dr. C. Wrede}

Interdisziplinäres Notfallzentrum, HELIOS Klinikum Berlin-Buch

Berlin

christian.wrede@helios-kliniken.de

Interessenkonflikt. B.W. Böttiger und C. Wrede geben an, dass kein Interessenkonflikt besteht. C. Waydhas: Mitgliedschaft in der DIVI, DGCH, DGU, ESICM, AAST.

\section{Neue Leitlinie für sinnvollen Antibiotika-Einsatz}

Der Anteil der Erreger, die gegen alle Breitspektrum-Antibiotika unempfindlich sind, steigt: In den letzten fünf Jahren um 50 bis 200 Prozent, laut Daten der Weltgesundheitsorganisation (WHO).

Eine neue Leitlinie der Arbeitsgemeinschaft wissenschaftlich-medizinischer Fachgesellschaften soll nun die Verbreitung resistenter Keime aufhalten. Sie gibt Empfehlungen, die in Ländern wie den USA, den Niederlanden oder Schweden längst Standard sind. Grundlage ist die Bildung interdisziplinärer Antibiotic Stewardship (ABS) Teams. Die Teams aus Infektiologen, Fachapothekern sowie Fachärzten für Mikrobiologie und Hygieneverantwortlichen sollen Krankenhäuser im sachgemäßen Umgang mit Antibiotika unterstützen. So sollen sie lokal umsetzbare Leitlinien zum Antibiotikaeinsatz im Haus erstellen und das Krankenhauspersonal aufklären und fortbilden. Darüber hinaus erheben die Teams Daten und Statistiken zum Antibiotikaverbrauch sowie zu Infektionen und Resistenzentwicklungen in deutschen Kliniken. Zu den Strategien der Resistenzbekämpfung gehören eine verkürzte Therapiedauer, eine optimierte Dosis und eine frühzeitige erregerspezifische Behandlung.

Quelle: 12. Kongress für Infektionskrankheiten und Tropenmedizin, www.KIT2014.de 